\title{
Seasonal Biodiversity Assessment of Benthic Macroinvertebrate of Asejire Reservoir, Southwest Nigeria
}

\author{
Godwin Irabor Asibor ${ }^{1}$ \\ ${ }^{1}$ Department of Environmental Sciences, College of Science, Federal University of Petroleum Resources, Effurun, \\ Nigeria \\ Correspondence: Godwin Irabor Asibor, Department of Environmental Sciences, College of Science, Federal \\ University of Petroleum Resources, P.M.B. 1221, Effurun, Delta State, Nigeria. E-mail: \\ asibor.godwin@fupre.edu.ng
}

Received: December 10, 2014 Accepted: December 29, 2014 Online Published: March 30, 2015

doi:10.5539/jsd.v8n2p257

URL: http://dx.doi.org/10.5539/jsd.v8n2p257

\begin{abstract}
The benthic macro-invertebrate fauna of Asejire Reservoir, Southwest Nigeria were investigated for two years (2004 - 2006) covering both the rainy and dry seasons. The major objective of the study was to provide baseline information on aspects of the biology (taxonomic composition, occurrence, distribution and abundance) of the benthic macro-invertebrates, characteristics of the reservoir sediment.

A total of twenty sampling stations were established to represent the various sections and regime of the lake. Sediment samples were collected from each station at bimonthly intervals using an improvised Van-veen grab. Altogether a total of three hundred and twenty composite samples were collected and analysed and assessed.

The benthic macro-invertebrates comprised twenty-eight species in the class Insecta (13 taxa), Gastropoda ( 9 taxa), Bivalvia (2 taxa) while Arachnida, Malacostraca, Hirudinea and Gordiadea were made of one species each. Altogether a total abundance of 364,351 individual macro-invertebratess species were collected from the bottom sediment. The dominant species were Potadomamoerch, P. freethi and Melanoidestubaculata with a population of 119,985, 66,660 and 32,768 individual taxawhile the least occurring taxa were Sphaeriumsp and Hydracarinasp with a population of 550 and 1,350 individual. Considerable close association $(\mathrm{p}<0.05)$ were found among many of the macro-invertebrates species. Species abundance was higher in the dry season $(46 \pm 6.5$ organism $\left.\mathrm{m}^{-2}\right)$ than in the rainy season $\left(36 \pm 6.0\right.$ organism $\left.\mathrm{m}^{-2}\right)$ but the difference between the two seasons was not significant $(\mathrm{p}>0.05)$. More species were found in the littoral than in the open water region of the reservoir and the difference between the two regions was significant $(\mathrm{p}<0.05)$. The differences in the abundance of species in the three reaches were also significant $(\mathrm{p}<0.05)$. In conclusion, on the basis of benthic macro-invertebrate taxa composition and abundance, Asejire Reservoir can be inferred to be rich in fauna composition and therefore fairly clean and unpolluted.
\end{abstract}

Keywords: benthic macroinvertebrates, reservoir, biodiversity assessment, Asejire Reservoir, Osun River

\section{Introduction}

Reservoirs are artificial water bodies whose dynamics and structures present a pattern of organization midway between those of rivers and lakes (Callisto et al., 2005). The ecological processes in these ecosystems are much more complex and variable than those found in natural lakes. Reservoirs are subject to distinguishable influences of the physical, chemical, and biological components of their tributaries (Torloni, 1994), as well as those caused by the principal land uses in the drainage basins.

The transformation from a lotic to a lentic system entail by depth increase due to river damming drastically alters the physical (e.g., subaquatic radiation, light, and temperature), chemical (dissolved oxygen and nutrient concentrations), and biological water characteristics (structure and aquatic community distribution patterns). Remains of chemical fertilizers coupled with industrial effluents and untreated sewage coming from the drainage basin cause profound modifications in most reservoirs. Among these changes are increases in trophic status and aquatic macrophyte growth, and sedimentation-rate modifications (Tundisi \& Straškraba, 1999).

According to Rosenberg et al. (2000), river diversion and large dams have contributed substantially to fishery destruction, species extinction, overall loss of ecosystem function that is crucial for humans, with results such as 
water-borne disease increase. This has also substantially caused a reduced use in sustaining river systems. Since 1950 the number of large dams (over 15 meters in height) has increased from less than 6,000 worldwide to over 40,000, thus converting many lotic systems into lentic ones (Johnson, et al., 2001). This has created an extensive habitat fragmentation in nearly $60 \%$ of the major river basins.

Within the aquatic communities, benthic macroinvertebrates represent one of the groups most affected by reservoir construction. These organisms inhabit river, lake, and reservoir bottoms, and their distribution is directly related to food availability and quantity, sediment type, substrate and water quality.

Data on macrobenthic community distribution and structure have been used in ecological monitoring programs, and is an important ecological tool to describe spatial and temporal changes (Leal \& Esteves, 1999). Depending on the distance between the dams along the river, reservoir systems have the potential to increase river impoundment effects on aquatic organism composition and distribution.

A change in the richness/diversity of benthic macroinvertebrates is to be expected along the reaches and sections, in accordance with the use of the drainage basin. Particularly considering the reservoir cascade formed by the large reservoirs in the Osun River, this study had as its major objective to evaluate macroinvertebrate diversity and also differences between the regions and reaches, while using these organisms as water-quality bioindicators.

\section{Study Area}

Asejire Reservoir is a manmade lake that was created in November 1970 by the impoundment of River Osun and officially opened in 1972 . River Osun catchment basin extends from longitudes $003^{\circ} 55^{\prime} \mathrm{E}$ to $005^{\circ} 05^{\prime} \mathrm{E}$ and latitudes $06^{0} 35^{\prime} \mathrm{N}$ to $08^{0} 20^{\prime} \mathrm{N}$. However, the catchment area of Asejire Reservoir is from longitudes $004^{0}$ $07^{\prime} 017^{\prime \prime} \mathrm{E}$ to $004^{\circ} 08^{\prime} 925^{\prime \prime} \mathrm{E}$ and in length from latitudes $07^{\circ} 21^{\prime} 48^{\prime \prime} \mathrm{N}$ and $07^{\circ} 26^{\prime} 84^{\prime \prime} \mathrm{N}$ (Figure 1). River Osun is one of the series of West African rivers which do not drain into Niger River system but discharge into coastal lagoons and creeks bordering the Atlantic Ocean. The lake is Y-shaped with two unequal arms of the Y (longer one is River Osun, while the shorter one is River Oba) (Ayodele, 1979). It was primarily created to supply domestic and industrial water, although some ancillary benefits such as fishing activities have also emerged (Asibor, 2008). The reservoir receives the bulk of its water input from two rivers, Rivers Osun and its main tributary River Oba. The area is typified by an admixture of savanna and high forest trees with palm, indicative of secondary rainforest vegetation. In addition there are numerous low hills with very gentle slopes, which effectively protect and shade the lake.

From the data supplied by the Oyo State Water Corporation of Nigeria, the catchment area of the dam is 7,800 $\mathrm{km}^{2}$ and the impounded area is $23.42 \mathrm{~km}^{2}$ (2,342 hectares). The dam has a normal pool elevation (water level) of $150 \mathrm{~m}$ and maximum flood elevation of $152.4 \mathrm{~m}$. The surface area of the reservoir is about $24 \mathrm{~km}^{2}$. Its gross storage capacity is approximately 7,403.4 million litres per day while its discharge capacity is 136.26 million litres per day (Ayodele, 1979) with maximum water capacity of about $675 \mathrm{~m}^{3}$.

Fishing activity is prominent in the reservoir. With the aforementioned enormous significance of the reservoir, no detailed scientific investigation has been carried out on the macro invertebrate benthic fauna of the reservoir which is an important biotic component of the food web and fishery of the reservoir. 


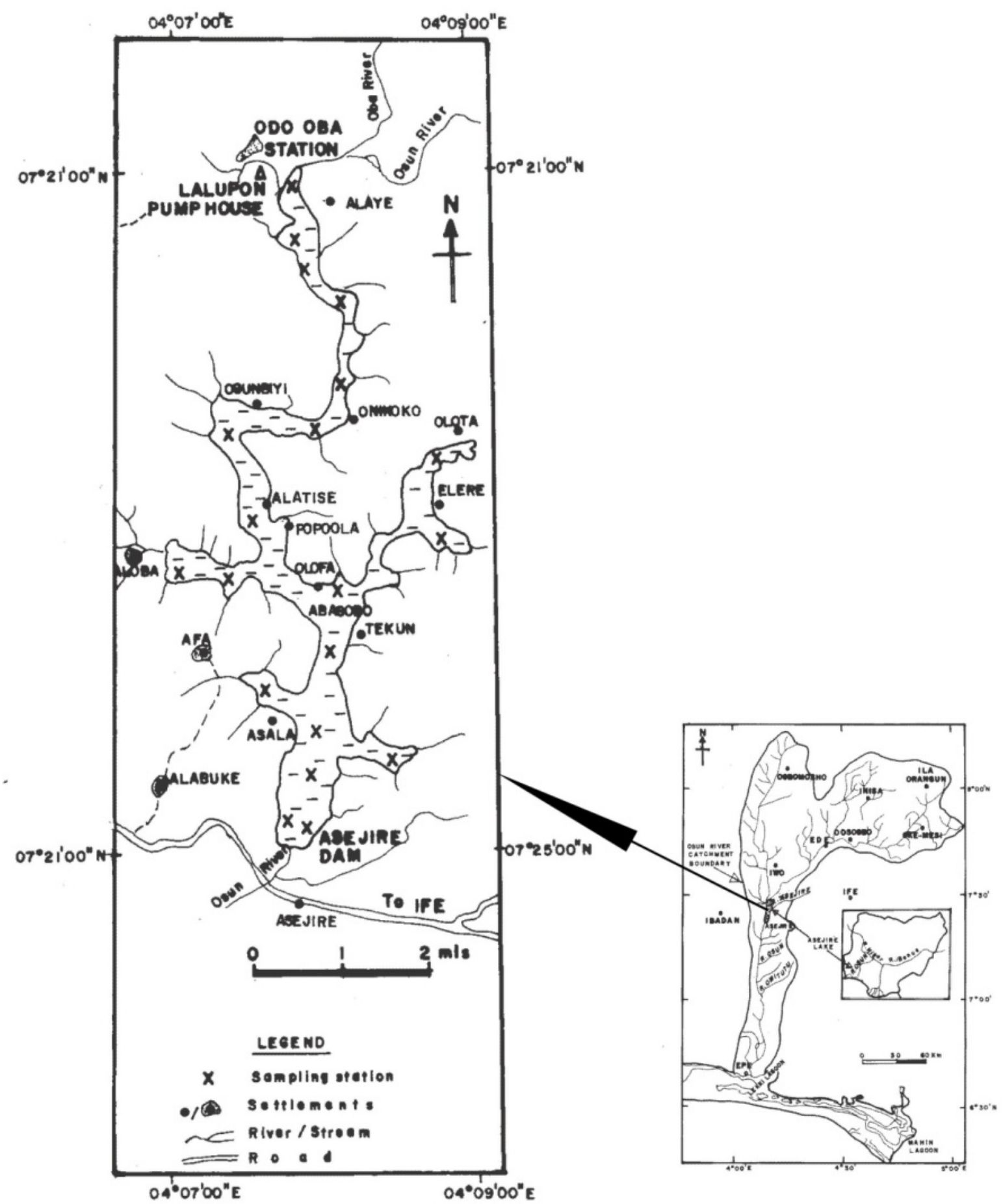

Figure 1. Map of Osun River Basin showing Asejire Reservoir, catchment basin and sampling locations

\section{Material and Methods}

Sampling was carried out aboard a hand-dug canoe every two months for two annual cycles (June 2004 to December 2006) using a improvised Van Veen grab sampler of $0.04 \mathrm{~m}^{2}(0.2 \mathrm{~m} \mathrm{X} 0.2 \mathrm{~m})$ for benthic fauna. The reservoir was divided into three sections (lower reach, mid-basin and upper reach), with an established 20 stations. Information on the grid locations of the stations and depth range is presented in Table 1. Grab samples were taken at each of the sampling sites, washed using a $0.5 \mathrm{~mm}$ mesh sieve, after which it was fixed with buffered 
$40 \%$ formaline. Samples of the periphyticmacrofauna on rocky substrates were collected by washing them directly into the sieve. In all a total of three hundred and twenty samples were collected for this study.

In the laboratory, the samples were washed using $0.125 \mathrm{~mm}$ mesh sieves, then sorted and identified under a stereomicroscope. The identification of the benthic macro-invertebrates collected in the study were based mainly on the keys provided by Brown (1980), Madsen (1985), Schneider (1990), Bouchard (2004) and Verma (2006). Description of specimens of taxa was based on scale drawings, photographs and/or microphotography of parts. The taxa richness, diversity and evenness indices were calculated using the Shannon-Wiener index.

All the statistical analyses were carried out using the Palaeotological Statistics (Hammer et al., 2003), Statistical Package for Social Sciences (SPSS) Software package for biological data analysis and Statistical Ecology (Ludwig \& Reynolds, 1988).

Table 1. Summary of the investigated sampling stations

\begin{tabular}{|c|c|c|c|c|c|c|c|c|c|}
\hline \multirow{2}{*}{$\begin{array}{l}\text { Sampling } \\
\text { Stn No. }\end{array}$} & \multicolumn{4}{|c|}{ Grid location } & \multirow{2}{*}{$\begin{array}{lr}\text { Distance } & \text { from } \\
\text { Dam } & \text { axis } \\
(\mathrm{Km}) & \end{array}$} & \multirow{2}{*}{$\begin{array}{l}\text { Depth } \\
\text { Range (m) }\end{array}$} & \multirow{2}{*}{$\begin{array}{c}\text { Mean } \pm \text { std } \\
\text { (m) }\end{array}$} & \multicolumn{2}{|c|}{ Classification } \\
\hline & $\begin{array}{l}\text { Lati } \\
\text { (N) }\end{array}$ & & $\begin{array}{l}\text { Longi } \\
\text { (E) }\end{array}$ & & & & & Reach & Region \\
\hline 1 & $07^{0}$ & $21^{\prime}$ & $004^{0}$ & $08^{\prime}$ & 0.28 & $15.2-16.4$ & $15.66 \pm 0.56$ & Upper & OWR \\
\hline 2 & $07^{0}$ & 21 & $004^{0}$ & $08^{\prime}$ & 0.24 & $14.6-16.0$ & $15.82 \pm 1.56$ & Upper & OWR \\
\hline 3 & $07^{0}$ & 22 & $004^{0}$ & $08^{\prime}$ & 1.23 & $15.2-16.8$ & $15.98 \pm 1.26$ & Upper & OWR \\
\hline 4 & $07^{0}$ & 22 & $004^{0}$ & $08^{\prime}$ & 2.37 & $10.2-11.0$ & $10.84 \pm 0.52$ & Upper & OWR \\
\hline 5 & $07^{0}$ & 22 & $004^{0}$ & $08^{\prime}$ & 3.22 & $6.5-8.2$ & $7.78 \pm 2.16$ & Upper & Littoral \\
\hline 6 & $07^{0}$ & 23, & $004^{0}$ & $08^{\prime}$ & 2.46 & $3.5-5.0$ & $4.22 \pm 2.16$ & Upper & Littoral \\
\hline 7 & $07^{0}$ & 23, & $004^{0}$ & $08^{\prime}$ & 3.69 & $4.2-4.8$ & $4.42 \pm 0.86$ & Mid-Basin & Littoral \\
\hline 8 & $07^{0}$ & 23, & $004^{0}$ & $08^{\prime}$ & 4.73 & $8.4-10.6$ & $9.64 \pm 0.86$ & Mid-Basin & OWR \\
\hline 9 & $07^{0}$ & 23, & $004^{0}$ & $08^{\prime}$ & 5.96 & $7.4-8.6$ & $8.08 \pm 0.24$ & Mid-Basin & OWR \\
\hline 10 & $07^{0}$ & 24 & $004^{0}$ & $08^{\prime}$ & 6.34 & $11.2-12.0$ & $11.48 \pm 0.24$ & Mid-Basin & Littoral \\
\hline 11 & $07^{0}$ & 24 & $004^{0}$ & $07^{\prime}$ & 7.86 & $3.6-4.8$ & $4.28 \pm 0.46$ & Mid-Basin & Littoral \\
\hline 12 & $07^{0}$ & 24 & $004^{0}$ & $07^{\prime}$ & 5.02 & $6.6-7.8$ & $7.28 \pm 0.46$ & Mid-Basin & Littoral \\
\hline 13 & $07^{0}$ & $24^{\prime}$ & $004^{0}$ & $08^{\prime}$ & 5.49 & $3.8-4.5$ & $4.22 \pm 0.96$ & Mid-Basin & Littoral \\
\hline 14 & $07^{0}$ & 24 & $004^{0}$ & $08^{\prime}$ & 8.05 & $2.0-4.2$ & $2.77 \pm 0.25$ & Lower & Littoral \\
\hline 15 & $07^{0}$ & $24^{\prime}$ & $004^{0}$ & $08^{\prime}$ & 7.86 & $2.9-5.8$ & $4.02 \pm 1.16$ & Lower & OWR \\
\hline 16 & $07^{0}$ & 25, & $004^{0}$ & $08^{\prime}$ & 8.71 & $6.8-8.2$ & $7.28 \pm 0.42$ & Lower & OWR \\
\hline 17 & $07^{0}$ & 25, & $004^{0}$ & $08^{\prime}$ & 10.13 & $6.8-7.8$ & $7.48 \pm 0.46$ & Lower & OWR \\
\hline 18 & $07^{0}$ & 25, & $004^{0}$ & $08^{\prime}$ & 10.51 & $6.4-7.2$ & $6.84 \pm 0.56$ & Lower & OWR \\
\hline 19 & $07^{0}$ & $26^{\prime}$ & $004^{0}$ & $08^{\prime}$ & 11.36 & $2.2-5.4$ & $3.49 \pm 1.06$ & Lower & Littoral \\
\hline 20 & $07^{0}$ & $26^{\prime}$ & $004^{0}$ & $08^{\prime}$ & 12.21 & $2.6-4.4$ & $3.28 \pm 0.84$ & Lower & Littoral \\
\hline
\end{tabular}

OWR $=$ Open Water Region

\section{Results}

\subsection{Structure and Composition of the Benthic Macroinvertebrate Communities}

A total of twenty-eight benthic macro-invertebrate animal taxa were recorded from Asejire Reservoir sediment samples. The fauna is made up of thirteen taxa of molluscs and insects, and one taxa each of crustacean and annelida.

Information on the ecotype differentiation, general distribution and seasonal abundance of recorded macro-invertebrate taxa of Asejire Reservoir is given in Tables 2, 3, 4, 5 and 6. The benthic macro-invertebrate taxa were found distributed across all habitats and were highly interspersed or mixed up between the different ecotypes in the three reaches and the two regions of the reservoir. Many known benthic littoral taxa Odonata (Sympetrumsp and Epicordulia), Ephemeroptera (Cloeondipterum and Caenissp) and Hemiptera (Belostomasp) 
were also found in the open water region, while also some known open water taxa like Dipteran (Ablablemyiasp, Simuliumdamnosum and Chaoborussp) were found in the sediment littoral sections of the reservoir.

There were twelve dominant occurring benthic macroinvertebrate taxa (i.e. occurring most frequently) in the reservoir. Potadomafreethi, P. moerchi and Melanoidestubaculata were the three most occurring species (found in all the twenty sampling locations) throughout the sampling period. They were closely followed by SimuliumdamnosumandChaoborus taxa $(90 \%$ occurrence in the locations). The least occurring taxa was Hydracarinasp (10\%) followed by Hydrometrasp, Macrobrachiummacrobrachium, Lymnaeanatalensis, Bulinusglobosus, Gyraulusdeflectus and Hirudosp ( $15 \%$ occurrence). The most abundant taxa was Potadomamoerchi (119,985 organism $\left.\mathrm{m}^{-3}\right)$ followed by Potadomafreethi $\left(32,768\right.$ organism $\left.\mathrm{m}^{-2}\right)$ and Melanoidestubaculata $\left(66,660\right.$ organism $\left.\mathrm{m}^{-2}\right)$. The least abundant taxa was Sphaeriumsp $\left(550\right.$ organismm $\left.^{-2}\right)$, followed by Hydracarinasp $\left(1,350\right.$ organism $\left.\mathrm{m}^{-2}\right)$ and Gyraulusdeflectus $\left(1,375\right.$ organism $\left.\mathrm{m}^{-2}\right)$ as shown in the calculated mean abundance and frequency occurrence of the respective taxa in Table 2.

Taxa distribution and abundance were more pronounced in Stations 6, 19 and 5. Out of the twenty-eight taxa recorded during the sampling period, twenty-seven taxa were recorded in Station 6 while Stations 19 and 5 had twenty-six and twenty-three taxa respectively. Station 9 had the least number of taxa (6) followed by Stations 1 and 8 respectively with just seven taxa found in them. Taxa abundance followed similar trend with taxa distribution. Highest taxa abundance was recorded in Station $6\left(41,275\right.$ organism m$\left.^{-2}\right)$, while Stations 5 and 19 have 30,358 organism $\mathrm{m}^{-2}$ and 29,890 organism $\mathrm{m}^{-2}$ respectively. The least abundant station was Stations $8(4,208$ organism $\left.\mathrm{m}^{-2}\right), 15\left(7,575\right.$ organism $\left.\mathrm{m}^{-2}\right)$ and $2\left(7,852\right.$ organism $\left.\mathrm{m}^{-2}\right)$.

\subsection{Seasonal Variations in Occurrence}

From the seasonal variations in the population density of the benthic macro-invertebrate taxa, it can be seen that most of the taxa had their population restricted to certain time of the year and this resulted to different and multi peaks in taxa abundance. The differences between the two seasons in all the taxa identified are shown in Table 2 . Benthic macro-invertebrate taxa were generally more abundant in the dry season than in the rainy season, with the exception of only six taxa (Baetiscasp, Caenissp, Ablablesmyiasp, Hydracarinasp, Mutelasp and Paragordiussp). Out of the twenty-eight taxa, only Potadomafreethi and P. moerchi showed significant difference $(\mathrm{p}<0.05)$ in abundance over the two seasons of the annual cycle. 
Table 2. Temporal variation in the abundance of total bemthicmacroinvertebrates (organism/M2) in Asejire Reservoir, June 2004 - December 2006

\begin{tabular}{|c|c|c|c|c|c|}
\hline Class & $\mathbf{S} / \mathbf{N}$ & Organism & Total Abun. & Total Occu. & $\%$ Freq. Occur. \\
\hline \multirow{13}{*}{ Insecta } & 1 & Sympetrumsp & 8450 & 11 & 55 \\
\hline & 2 & Epicorduliasp & 6800 & 12 & 60 \\
\hline & 3 & Enallagmadeserti & 5000 & 9 & 45 \\
\hline & 4 & Ishnurasp & 5600 & 9 & 45 \\
\hline & 5 & Cloeondipterum & 7375 & 11 & 55 \\
\hline & 6 & Baetiscasp & 5402 & 9 & 45 \\
\hline & 7 & Caenissp & 4900 & 10 & 50 \\
\hline & 8 & Belostomasp & 6677 & 11 & 55 \\
\hline & 9 & Renatrasp & 5375 & 9 & 45 \\
\hline & 10 & Hydrometrasp & 1525 & 3 & 15 \\
\hline & 11 & Simuliumdamnosum & 23375 & 18 & 90 \\
\hline & 12 & Ablabesmyiasp & 18050 & 17 & 85 \\
\hline & 13 & Chaoborussp & 13339 & 18 & 90 \\
\hline Arachnida & 14 & Hydracarinasp & 1350 & 2 & 10 \\
\hline Malacostraca & 15 & Macrobrachiummacrobrachium & 1500 & 3 & 15 \\
\hline \multirow{9}{*}{ Gastropod } & 16 & Lymnaeanatalensis & 1575 & 3 & 15 \\
\hline & 17 & Biomphalariapfeifferi & 9566 & 10 & 50 \\
\hline & 18 & Bulinusglobosus & 3200 & 3 & 15 \\
\hline & 19 & Gyraulusdeflectus & 1375 & 3 & 15 \\
\hline & 20 & Physagyrina & 1800 & 6 & 30 \\
\hline & 21 & Potadomafreethi & 66660 & 20 & 100 \\
\hline & 22 & Potadomamoerchi & 119985 & 20 & 100 \\
\hline & 23 & Melanoidestubaculata & 32768 & 20 & 100 \\
\hline & 24 & Pilaovata & 2525 & 6 & 30 \\
\hline \multirow{2}{*}{ Bivalvia } & 25 & Sphaerium simile & 550 & 2 & 10 \\
\hline & 26 & Mutelasp & 2852 & 6 & 30 \\
\hline Hirudinea & 27 & Hirudosp & 1625 & 3 & 15 \\
\hline Gordiodea & 28 & Paragordiussp & 5152 & 14 & 70 \\
\hline
\end{tabular}

Table 3. Temporal variation in the abundance of total bemthicmacroinvertebrates (organism/M2) in Asejire Reservoir, June 2004 - December 2006

\begin{tabular}{|c|c|c|c|c|c|c|c|c|c|c|c|c|c|c|c|c|c|c|c|c|c|}
\hline & & \multicolumn{20}{|c|}{ STATION } \\
\hline & & 1 & 2 & 3 & 4 & 5 & 6 & 7 & 8 & 9 & 10 & 11 & 12 & 13 & 14 & 15 & 16 & 17 & 18 & 19 & 20 \\
\hline & Taxa & 7 & 9 & 11 & 9 & 23 & 27 & 9 & 7 & 6 & 10 & 15 & 18 & 14 & 18 & 8 & 8 & 13 & 20 & 26 & 15 \\
\hline & Individuals & 11354 & 9452 & 7852 & 10250 & 30358 & 41275 & 15975 & 10850 & 4208 & 14650 & 22625 & 24933 & 25602 & 28000 & 7575 & 8400 & 11827 & 25575 & 29890 & 23700 \\
\hline \multirow{5}{*}{$\begin{array}{l}\text { DIVERSITY } \\
\text { INDICES }\end{array}$} & Shannon & & & & & & & & & & & & & & & & & & & & \\
\hline & & 1.72 & 1.87 & 2.15 & 1.50 & 2.13 & 2.57 & 1.91 & 1.77 & 1.27 & 1.62 & 1.68 & 2.39 & 1.65 & 2.13 & 1.87 & 1.88 & 1.71 & 2.33 & 2.73 & 2.32 \\
\hline & $\begin{array}{l}\text { Simpson } \\
\text { ind } x\end{array}$ & 0.79 & 0.83 & 0.87 & 0.64 & 0.77 & 0.86 & 0.82 & 0.81 & 0.62 & 0.69 & 0.72 & 0.87 & 0.72 & 0.81 & 0.83 & 0.83 & 0.70 & 0.84 & 0.88 & 0.87 \\
\hline & Menhinick & 0.07 & 0.09 & 0.12 & 0.09 & 0.13 & 0.13 & 0.07 & 0.07 & 0.09 & 0.08 & 0.10 & 0.11 & 0.09 & 0.11 & 0.09 & 0.09 & 0.12 & 0.13 & 0.15 & 0.10 \\
\hline & Margalef & 0.64 & 0.87 & 1.12 & 0.87 & 2.13 & 2.45 & 0.83 & 0.65 & 0.60 & 0.94 & 1.40 & 1.68 & 1.28 & 1.66 & 0.78 & 0.77 & 1.28 & 1.87 & 2.43 & 1.39 \\
\hline
\end{tabular}


Table 4. Analysis of variance of relative abundance of benthic macroinvertebrates (organism/ $\mathrm{m} 2$ ) reflecting the total seasonal variation in Asejire Reservoir

\begin{tabular}{|c|c|c|c|c|c|c|c|}
\hline \multirow{3}{*}{ SN } & \multirow{3}{*}{ Species Name } & \multicolumn{4}{|c|}{ SEASON } & \multicolumn{2}{|c|}{$\begin{array}{c}\text { One-Way ANOVA }(\mathrm{df}=1 \& \\
318)\end{array}$} \\
\hline & & \multicolumn{2}{|c|}{$\mathrm{RS}(\mathrm{n}=160)$} & \multicolumn{2}{|c|}{$\mathrm{DS}(\mathrm{n}=160)$} & \multirow[t]{2}{*}{ f-values } & \multirow[t]{2}{*}{ p-values } \\
\hline & & $\mathrm{x}$ & s.e.m. & $\mathrm{x}$ & s.e.m. & & \\
\hline 1 & Sympetrumsp & 602 & 34.4 & 719 & 34.9 & 0.773600 & 0.37980 \\
\hline 2 & Epicorduliasp & 500 & 35.2 & 563 & 35.4 & 0.217800 & 0.64100 \\
\hline 3 & $\underline{\text { Enallagmadeserti }}$ & 320 & 27.5 & 461 & 27.5 & 3.472000 & 0.06334 \\
\hline 4 & Ishnurasp & 398 & 29.4 & 477 & 28.4 & 0.741700 & 0.38980 \\
\hline 5 & $\underline{\text { Cloeondipterum }}$ & 656 & 33.8 & 496 & 28.8 & 1.916000 & 0.16730 \\
\hline 6 & Baetiscasp & 430 & 29.7 & 414 & 29.0 & 0.035340 & 0.85100 \\
\hline 7 & $\underline{\text { Caenissp }}$ & 422 & 32.4 & 344 & 30.6 & 0.572200 & 0.45000 \\
\hline 8 & Belostomasp & 465 & 28.3 & 578 & 28.3 & 1.790000 & 0.18190 \\
\hline 9 & Renatrasp & 387 & 27.8 & 453 & 28.1 & 0.776900 & 0.37870 \\
\hline 10 & Hydrometrasp & 94 & 17.3 & 145 & 21.1 & 1.488000 & 0.22340 \\
\hline 11 & $\underline{\text { Simuliumdamnosum }}$ & 1766 & 30.1 & 1887 & 31.4 & 0.367600 & 0.54480 \\
\hline 12 & Ablabesmyiasp & 1453 & 31.3 & 1367 & 30.5 & 0.204700 & 0.65120 \\
\hline 13 & Chaoborussp & 962 & 37.2 & 1122 & 32.2 & 0.903500 & 0.34260 \\
\hline 14 & Hydracarinasp & 117 & 19.7 & 94 & 17.3 & 0.243700 & 0.62190 \\
\hline 15 & $\underline{\text { Macrobrachium }}$ & 102 & 18.9 & 133 & 19.8 & 0.487200 & 0.48570 \\
\hline 16 & Lymnaeanatalensis & 94 & 17.3 & 152 & 22.4 & 1.776000 & 0.18360 \\
\hline 17 & Biomphalariapfeifferi & 665 & 30.1 & 829 & 30.3 & 2.334000 & 0.12760 \\
\hline 18 & Bulinusglobosus & 109 & 20.0 & 141 & 20.7 & 0.562800 & 0.43370 \\
\hline 19 & $\underline{\text { Gyraulusdeflectus }}$ & 86 & 16.0 & 129 & 20.9 & 1.213000 & 0.27170 \\
\hline 20 & Physagyrina & 109 & 20.0 & 172 & 22.6 & 1.942000 & 0.16440 \\
\hline 21 & $\underline{\text { Potadomafreethi }}$ & 4338 & 60.8 & 6078 & 68.3 & 6.340000 & $0.0123 *$ \\
\hline 22 & $\underline{\text { Potadomamoerchi }}$ & 3923 & 57.0 & 5523 & 65.1 & 6.456000 & $0.01153 *$ \\
\hline 23 & $\underline{\text { Melanoidestubaculata }}$ & 2370 & 36.9 & 2750 & 39.6 & 2.229000 & 0.13650 \\
\hline 24 & $\underline{\text { Pilaovata }}$ & 188 & 22.9 & 207 & 24.8 & 0.134300 & 0.71430 \\
\hline 25 & Sphaeriumsimile & 23 & 9.1 & 63 & 14.5 & 2.747000 & 0.09842 \\
\hline 26 & Mutelasp & 227 & 24.0 & 219 & 23.2 & 0.025390 & 0.87350 \\
\hline 27 & $\underline{\text { Hirudosp }}$ & 121 & 19.8 & 133 & 19.5 & 0.08002 & 0.77750 \\
\hline 28 & $\underline{\text { Paragordiussp }}$ & 414 & 32.3 & 391 & 30.3 & 0.05083 & 0.82120 \\
\hline
\end{tabular}

* Significant at $\mathrm{p} \leq 0.05$

$* *$ Significant at $\mathrm{p} \leq 0.01$

$* * *$ Significant at $\mathrm{p} \leq 0.001$ 
Table 5. Analysis of variance reflecting species abundance (organism/M2) in the two regions of Asejire Reservoir

\begin{tabular}{|c|c|c|c|c|c|c|c|}
\hline \multirow{3}{*}{ SN } & \multirow{3}{*}{ Species Name } & \multicolumn{4}{|c|}{ REGION } & \multicolumn{2}{|c|}{ One-Way ANOVA $(\mathrm{df}=1 \& 318$} \\
\hline & & \multicolumn{2}{|c|}{ Open $(n=160)$} & \multicolumn{2}{|c|}{ Littoral $(\mathrm{n}=160)$} & \multirow[t]{2}{*}{ f-values } & \multirow[t]{2}{*}{ p-values } \\
\hline & & $\mathrm{x}$ & s.e.m. & $\mathrm{x}$ & s.e.m. & & \\
\hline 1 & Sympetrumsp & 47 & 13.23 & 1273 & 26.60 & 115.2 & $3.93 \mathrm{E}-23 * * *$ \\
\hline 2 & Epicorduliasp & 39 & 12.78 & 1023 & 35.98 & 65.04 & $1.51 \mathrm{E}-14 * * *$ \\
\hline 3 & $\underline{\text { Enallagmadeserti }}$ & 0 & 0.00 & 781 & 24.55 & 159 & $7.72 \mathrm{E}-30 * * *$ \\
\hline 4 & Ishnurasp & 0 & 0.00 & 875 & 24.01 & 131.1 & $1.21 \mathrm{E}-25 * * *$ \\
\hline 5 & $\underline{\text { Cloeondipterum }}$ & 94 & 18.28 & 1059 & 22.73 & 88.33 & $1.13 \mathrm{E}-18 * * *$ \\
\hline 6 & Baetiscasp & 0 & 0.00 & 844 & 24.91 & 144 & $1.28 \mathrm{E}-27 * * *$ \\
\hline 7 & Caenissp & 23 & 9.26 & 742 & 34.02 & 57.01 & 4.63E- $13 * * *$ \\
\hline 8 & Belostomasp & 235 & 24.80 & 809 & 27.45 & 53.01 & $2.62 \mathrm{E}-12 * * *$ \\
\hline 9 & $\underline{\text { Renatrasp }}$ & 0 & 0.00 & 840 & 22.38 & 203.2 & $5.48 \mathrm{E}-36 * * *$ \\
\hline 10 & Hydrometrasp & 0 & 0.00 & 238 & 25.42 & 36.34 & $4.58 \mathrm{E}-09 * * *$ \\
\hline 11 & $\underline{\text { Simuliumdamnosum }}$ & 2625 & 28.86 & 1027 & 24.57 & 79.99 & $3.17 \mathrm{E}-17 * * *$ \\
\hline 12 & Ablabesmyiasp & 2063 & 31.89 & 758 & 28.76 & 55.36 & $9.43 \mathrm{E}-13 * * *$ \\
\hline 13 & Chaoborussp & 1448 & 37.26 & 637 & 31.54 & 24.79 & $1.05 \mathrm{E}-06 * * *$ \\
\hline 14 & $\underline{\text { Hydracarinasp }}$ & 0 & 0.00 & 211 & 23.91 & 21.03 & $6.50 \mathrm{E}-06 * * *$ \\
\hline 15 & Macrobrachium & 0 & 0.00 & 234 & 25.78 & 29.94 & $9.06 \mathrm{E}-08 * * *$ \\
\hline 16 & $\underline{\text { Lymnaeanatalensis }}$ & 0 & 0.00 & 246 & 24.80 & 34.54 & $1.05 \mathrm{E}-08 * * *$ \\
\hline 17 & Biomphalariapfeifferi & 180 & 22.46 & 1315 & 22.68 & 170.4 & $1.78 \mathrm{E}-31 * * *$ \\
\hline 18 & Bulinusglobosus & 0 & 0.00 & 250 & 24.80 & 40.54 & $6.73 \mathrm{E}-10 * * *$ \\
\hline 19 & Gyraulusdeflectus & 0 & 0.00 & 215 & 23.63 & 33.37 & $1.82 \mathrm{E}-08 * * *$ \\
\hline 20 & Physagyrina & 0 & 0.00 & 281 & 25.44 & 44.56 & $1.10 \mathrm{E}-10 * * *$ \\
\hline 21 & $\underline{\text { Potadomafreethi }}$ & 1883 & 29.71 & 8533 & 63.86 & 126.9 & $5.31 \mathrm{E}-25 * * *$ \\
\hline 22 & $\underline{\text { Potadomamoerchi }}$ & 1766 & 27.43 & 7680 & 60.53 & 118.6 & $1.10 \mathrm{E}-23 * * *$ \\
\hline 23 & $\underline{\text { Melanoidestubaculata }}$ & 1430 & 24.84 & 3690 & 31.84 & 103.9 & $2.67 \mathrm{E}-21 * * *$ \\
\hline 24 & Pilaovata & 8 & 5.18 & 387 & 27.07 & 60.05 & $1.26 \mathrm{E}-13 * * *$ \\
\hline 25 & Sphaeriumsimile & 0 & 0.00 & 86 & 17.07 & 13.75 & $2.46 \mathrm{E}-04 * * *$ \\
\hline 26 & Mutelasp & 211 & 21.80 & 235 & 25.38 & 0.2171 & $6.42 \mathrm{E}-01$ \\
\hline 27 & $\underline{\text { Hirudosp }}$ & 0 & 0.00 & 254 & 24.80 & 42.58 & $2.67 \mathrm{E}-10 * * *$ \\
\hline 28 & Paragordiussp & 508 & 32.84 & 297 & 28.38 & 4.298 & $0.03895 *$ \\
\hline
\end{tabular}

* Significant at $\mathrm{p} \leq 0.05$

$* *$ Significant at $\mathrm{p} \leq 0.01$

$* * *$ Significant at $\mathrm{p} \leq 0.001$ 
Table 6. Analysis of variance reflecting species abundance (organism/M2) in the three reaches of Asejire Reservoir

\begin{tabular}{|c|c|c|c|c|c|c|c|c|c|}
\hline \multirow{3}{*}{$\mathrm{SN}$} & \multirow{3}{*}{ Species Name } & \multicolumn{6}{|c|}{ REACHES } & \multicolumn{2}{|c|}{$\begin{array}{c}\text { One-Way ANOVA }(\mathrm{df}=1 \& \\
317)\end{array}$} \\
\hline & & \multicolumn{2}{|c|}{ Upper $(n=96)$} & \multicolumn{2}{|c|}{ Mid (n=112) } & \multicolumn{2}{|c|}{ Lower $(\mathrm{n}=112)$} & \multirow[t]{2}{*}{ f-values } & \multirow[t]{2}{*}{ p-values } \\
\hline & & $\mathrm{x}$ & s.e.m. & $\mathrm{x}$ & s.e.m. & $\mathrm{x}$ & s.e.m. & & \\
\hline 1 & Sympetrumsp & 768 & 36.01 & 703 & 31.91 & 525 & 37.88 & 1.12 & 0.3275 \\
\hline 2 & Epicorduliasp & 938 & 38.43 & 446 & 33.03 & 268 & 29.60 & 11.68 & $1.276 \mathrm{E}-05 * * *$ \\
\hline 3 & Enallagmadeserti & 365 & 27.18 & 391 & 29.95 & 413 & 32.08 & 0.14 & $8.73 \mathrm{E}-01$ \\
\hline 4 & Ishnurasp & 586 & 29.50 & 424 & 25.82 & 324 & 28.90 & 3.88 & $0.02166 *$ \\
\hline 5 & $\underline{\text { Cloeondipterum }}$ & 814 & 33.44 & 513 & 27.10 & 435 & 31.83 & 4.28 & $0.0147 *$ \\
\hline 6 & Baetiscasp & 456 & 28.48 & 425 & 24.60 & 391 & 30.34 & 0.25 & 0.7769 \\
\hline 7 & $\underline{\text { Caenissp }}$ & 703 & 34.93 & 212 & 23.19 & 279 & 28.43 & 7.11 & $9.543 \mathrm{E}-04 * * *$ \\
\hline 8 & Belostomasp & 912 & 30.36 & 385 & 29.53 & 324 & 23.12 & 22.93 & $4.99 \mathrm{E}-10 * * *$ \\
\hline 9 & $\underline{\text { Renatrasp }}$ & 534 & 26.60 & 340 & 25.67 & 402 & 30.07 & 0.99 & 0.3735 \\
\hline 10 & $\underline{\text { Hydrometrasp }}$ & 143 & 21.14 & 11 & 7.37 & 206 & 18.84 & 0.65 & 0.5242 \\
\hline 11 & $\underline{\text { Simuliumdamnosum }}$ & 1341 & 21.64 & 1641 & 34.24 & 2427 & 34.58 & 11.41 & $1.635 \mathrm{E}-05 * * *$ \\
\hline 12 & $\underline{\text { Ablabesmyiasp }}$ & 951 & 32.58 & 1440 & 32.66 & 1775 & 33.65 & 6.43 & $1.82 \mathrm{E}-03 * *$ \\
\hline 13 & Chaoborussp & 781 & 34.30 & 1118 & 34.20 & 1190 & 37.68 & 2.15 & 0.1183 \\
\hline 14 & Hydracarinasp & 182 & 24.83 & 0 & 0.00 & 145 & 21.37 & 5.82 & $3.29 \mathrm{E}-03 * *$ \\
\hline 15 & $\underline{\text { Macrobrachium }}$ & 143 & 21.38 & 0 & 0.00 & 212 & 25.42 & 8.62 & $2.27 \mathrm{E}-04 * * *$ \\
\hline 16 & Lymnaeanatalensis & 169 & 22.79 & 0 & 0.00 & 206 & 22.86 & 9.19 & $1.31 \mathrm{E}-04 * * *$ \\
\hline 17 & Biomphalariapfeifferi & 1029 & 29.80 & 671 & 31.51 & 582 & 31.04 & 6.40 & $1.88 \mathrm{E}-03 * *$ \\
\hline 18 & Bulinusglobosus & 182 & 20.76 & 0 & 0.00 & 201 & 23.83 & 10.49 & $3.86 \mathrm{E}-05 * * *$ \\
\hline 19 & $\underline{\text { Gyraulusdeflectus }}$ & 156 & 21.03 & 0 & 0.00 & 173 & 22.81 & 8.73 & $2.04 \mathrm{E}-04 * * *$ \\
\hline 20 & Physagyrina & 195 & 21.97 & 11 & 7.37 & 223 & 25.91 & 9.68 & $8.27 \mathrm{E}-05 * * *$ \\
\hline 21 & $\underline{\text { Potadomafreethi }}$ & 4716 & 61.53 & 6127 & 67.83 & 4710 & 66.64 & 1.91 & $1.50 \mathrm{E}-01$ \\
\hline 22 & $\underline{\text { Potadomamoerchi }}$ & 4227 & 56.04 & 5407 & 71.10 & 4464 & 69.31 & 1.32 & $2.68 \mathrm{E}-01$ \\
\hline 23 & $\underline{\text { Melanoidestubaculata }}$ & 2427 & 34.29 & 3058 & 37.35 & 2176 & 42.50 & 4.57 & $1.11 \mathrm{E}-02 *$ \\
\hline 24 & $\underline{\text { Pilaovata }}$ & 391 & 26.86 & 22 & 8.77 & 206 & 21.89 & 17.48 & $6.26 \mathrm{E}-08 * * *$ \\
\hline 25 & $\underline{\text { Sphaeriumsimile }}$ & 78 & 15.73 & 0 & 0.00 & 56 & 12.95 & 4.02 & $0.01884 *$ \\
\hline 26 & $\underline{\text { Mutelasp }}$ & 254 & 23.79 & 0 & 0.00 & 419 & 26.16 & 28.25 & $5.07 \mathrm{E}-12 * * *$ \\
\hline 27 & $\underline{\text { Hirudosp }}$ & 182 & 23.95 & 0 & 0.00 & 206 & 16.78 & 11.03 & $2.33 \mathrm{E}-05 * * *$ \\
\hline 28 & $\underline{\text { Paragordiussp }}$ & 46 & 12.48 & 435 & 32.20 & 676 & 32.48 & 13.52 & $2.32 \mathrm{E}-06 * * *$ \\
\hline
\end{tabular}

* Significant at $\mathrm{p} \leq 0.05$

$* *$ Significant at $\mathrm{p} \leq 0.01$

$* * *$ Significant at $\mathrm{p} \leq 0.001$

\subsection{Relationship between the Reservoir Regions}

Table 5 shows taxa abundance in the two regions (littoral and open water regions). Twenty-eight taxa were found in the littoral region as opposed to only fifteen taxa found in the open water region. Out of these, twenty-four taxa were more abundant in the Littoral region, while only four taxa (Simuliumdamnosum, Ablablesmyiasp, Chaoborussp and Paragordiussp) were more abundant in the open water region than in the littoral region. Generally taxa were more abundant in the littoral region than in the open water region. 
Relationship between the two regions showed that taxa abundance was significantly different $(p<0.05)$ for twenty-seven taxa of the twenty-eight. It was only Mutelasp that did not show significant difference between the two regions.

\subsection{Relationship between the Reservoir Reaches}

The relationship between the three reaches of the reservoir in terms of taxa abundance is shown in Table 6 . The lower reach had the highest number of taxa (thirteen), followed by the upper reach (twelve) while the mid reach had the least number of taxa with just three taxa (Potadomafreethi, P. moerchi and Melanoidestubaculata). All the twenty-eight taxa were present in both the upper and lower reaches, while eight taxa (Hydracarinasp, Macrobrachiummacrobrachium, Lymnaeanatalensis, Bulinusglobosus, Gyraulusdeflectus, Sphaerium simile, Mutelasp, Hirudosp and Paragordiussp) were absent in the mid reach of the reservoir during the sampling period. Twenty out of the twenty-eight taxa showed significant differences $(p<0.05)$ in the taxa abundance over the three reaches of the reservoir.

\section{Discussion}

The benthic macro-invertebrate fauna of Asejire reservoir is broadly similar to that of other Nigerian and African inland waters including lake Kanji (Taiwo, 1983), Eleyele stream (Fagbola, 1989), Owena reservoir (Oke, 1989), Opa Reservoir (Nathaniel, 2001), River Galma (Adakole, 2003), Ibiekuma River (Edokpayi and Osimen, 2001) and Ikpoba River (Ogbeibu and Oribhabor, 2001) especially with respect to species composition. A total of twenty-eight benthic macro-invertebrate animal species made up of the following class were recorded in the reservoir: class Insecta (13 taxa), Gastropoda (9 taxa), Bivalvia (2 taxa) while Arachnida, Malacostraca, Hirudinea and Gordiadea were made of one taxon each. While the Insecta were made up of different larvae forms, the others were fully grown adults.

Though there is no established checklist of available benthic macroinvertebrate taxa of Nigerian inland waters (Nathaniel op cit), most of the recorded species have been identified by other workers. Some of these include the work of Okpalla (1961), who worked on Bulinusglobosus and concluded that this species has a wide distribution in the Northern and Western regions of Nigeria while Cowper (1963) concluded that Bulinusglobosus, Lymneanatalensis, Lanisteslibycus and Biomphalariapfeiferi are known to occur in most parts of Nigeria. Adewumi (1984) and Fagbola (1989) recorded Potadoma, moerchi in the river bottom of streams in Ile-Ife, while Aliu (2006) recorded P. moerchi,also in Opa river bottom of streams in Ile-Ife. The different larval forms of insects (Anisoptera and Zygoptera) have been recorded in Owena reservoir and lake Kanji (Bidwell and Clarke 1977) and in Owena Reservoir (Oke 1989). Nathaniel (2001) worked on Opa Reservoir in Ile-Ife and recorded seven species made up of Molluscs and Insects while Aliu (2006) worked on different streams flowing into Opa Reservoir and recorded twenty species belonging to Mollusca, Insecta and Annelida.

The dominant species in Asejire reservoir were Potodomafreethi, P. moerchi and Melanoidestubaculata all belonging to the phylum mollusca. This observation agrees with the recent work of Owojori (2004) and Aliu (2006) who worked on streams at Ile-Ife and recorded high abundance of Melanoidestubaculata, Bulinusglobosus, Potadormamoerchi, P freethi and Lymnaeanatalensis, while Oke (1989), Fagbola (1989) and Nathaniel (2001) recorded Biomphalaripfepferias the dominant species in Owena Reservoir, Ile-Ife streams and Opa Reservoir

The large number of Potodomafreethi and P. moerch, recorded in this study compare with other species collected was not surprising because it has been reported to be widely distributed in streams and rivers in West and Central Africa rainforest regions (Brown, 1980; Agbolade and Odaibo, 2004). While Potadomafreethi is commomnly found from Ivory Coast to lower Zaire, P. moerchi is present from Southeast Ghana to Nigeria (Mandahl-Barth, 1967 and Brown, 1994). Potadomafreethi showed strong association with rivers of clear, well oxygenated water flowing over rocks or gravel and lacking aquatic plants.

In comparison to the available studies of inland waters the macro-invertebrate benthic species recorded in Asejire reservoir can be considered to be poor in taxa distribution but rich in the total of individuals collected during the sampling period. For instance, in Asejire reservoir twenty-eight species comprising 364,351 individuals were recorded for the two year duration of the sampling period, while in Opa reservoir seven species comprising 378 individuals were recorded (Nathaniel, 2001), 12,076 individuals in Bindare and Galma Rivers (Adakole, 2003) and 11,063 individuals from Owena reservoir comprising of thirteen species (Oke, 1989). In Lake Kainji (the largest man-made lake in Nigeria) thirteen species comprising 23,261 individuals were recorded (Taiwo, 1983), forty-one taxa comprising of 4,614 individuals (Ogbeibu \& Oribhabor, 2001), eighty-nine taxa comprising 2,535 individuals (Edokpayi \& Osimen, 2001). 
According to Jayne and Joann (1999) an impoverished macro-invertebrate benthic fauna community could be attributed to the physico-chemical and geochemical nature of the sediment (fertility), water depth, sediment type, water current, sediment erosion due to intensive farming and clearing of ground cover which cause silting and adverse effect on the macro-invertebrate benthic community. From the available data on the Asejire Reservoir, it can be inferred that the reservoir is rich in organic carbon and other nutrients with slow currents and thick covering of its shoreline with vegetation. This provides a good breeding ground and source of food for the benthic organisms to thrive on. Another reason for the high number of individuals could be attributable to high frequency of sampling and the duration (two calendar years).

As seen from the available results (Tables 5) the bulk of the macro-invertebrate benthic animals in the reservoir occurred more in the littoral region than in the open water region. According to Cole, 1975) the littoral area is well lighted, with high dissolved oxygen. It also contains high abundance of nutrients like nitrogen and phosphorus with high growth of algae and other aquatic plants. Harold et al. (1998) observed that there is usually extensive growth of submerged or emergent plants in the littoral region, which provide protection from predators for variety of benthic macroinvetebrates and a good substrate for growth of epiphytic algae. The high variety of benthic macroinvetebrtaes recorded in Asejire reservoir also agrees with the work of Koester and Bryan (1989) who reported that it is in the fringing area (littoral region) that most the most abundant, widely distributed and diverse form of benthic macroinvertebratess occurs. The open water benthic region on the other hand is highly impoverished as a result of reduced light penetration, reduced number of micro-habitats and depleted dissolved oxygen leading to reduced environment and as a result of low redox potentials. The presence of high number of Simuliumdamnosum, Ablablesmyiasp, Chaoborussp and Paragordiussp in the region is not surprising. These are species that can withstand low disolved oxygen and a stressed environment. They are also used as indicator of a stressed environment. In Asejire Reservoir the area is covered by dense floating vegetation Ludwigia, Pistia and Eloidea weeds whose decomposition could influence food supply to the benthos and hence the benthic community. So, it is not surprising that benthic animals occurred more in the region than in the profundal region. The spread of benthic macroinvertebrates within the reaches was not well demarcated, but taxa were more in the upper and lower reaches than in the mid-reach.

\subsection{Seasonal Benthic Macroinvertebrate Distribution}

The results of seasonal abundance in invertebrate benthic macroinvertebrate are shown in Table 4. On the whole, macro-invertebrate benthic faunal population was higher in the dry season than in the rainy season in most of the species (twenty-one species out of the twenty-eight species) identified. This may be attributed to recorded seasonal variation in the physico-chemical parameters of the sediments. The different peaks observed in the population pattern reflect more organisms being recruited into the population. The distribution pattern of the different macro-invertebrate species in each month of sampling indicates the succession among the recorded species. The various peak patterns (single, two and multiple) observed in the seasonal period indicate their generation cycle. Multiple peaks suggest that macro-invertebrate benthic fauna species concerned has many generations during the annual cycle. The decline in population after each peak probably indicates the end of a generation and this varies with species and habitats. This may imply that the spatial variation in the population dynamics of the macro-invertebrate benthic species vary with habitats of the reservoir. This explanation had earlier been given by Mclachlam and Mclachlam, 1971, who reported that macro-invertebrate benthic fauna's population dynamics in the tropics may occur at any particular period of the year depending on the predominating set of physicochemical and biological conditions in the ecosystem. This is probably the basis of suggestion by Jayne and Joann (1999) that the dynamics in the population of the bottom dwelling benthic macro- invertebrate fauna within sub-habitats in a major habitat varies with variation in environmental factors of the sediments.

\section{Conclusion}

There were significant differences $(\mathrm{p}<0.05)$ between the three reaches of the reservoir in taxa abundance; a trend also observed in the regions of the reservoir, this is attributable to the abundance of light, shelter and food supply to these areas of the reservoir. The reservoir is considered to be rich in macro-invertebrate fauna when compared with some lakes, reservoirs and inland streams in Nigeria, Africa, Europe and America but impoverished in terms of taxa occurrence.

On the basis of benthic macro-invertebrate taxa composition and abundance, Asejire Reservoir can be inferred to be rich in fauna composition and therefore fairly clean and unpolluted as at the time the study was carried out. This is because of the low presence of discharges to the reservoir from anthropogenic sources. To maintain the pristine nature of the reservoir for sustainable use of the reservoir, there is need for continuous monitoring of not just the reservoir but also rivers and stream flowing into it. Industries and factories within the catchment area should be 
encouraged to treat and monitor effluent and wastewater before discharging them.

\section{References}

Adakole, J. A. (2003). Benthic macroinvertebrates as indicators of environmental quality of an urban stream, Zaria, Northern Nigeria. Journal of Aquatic Sciences, 18(2), 85-92.

Adewumi, C.O. (1984). Water extract of Tetrapleuratetrapleura: an effective molluscide for the control of Schistosomiasis in Nigeria. Journal of Animal Production Research, 4(1), 73-84.

Agbolade, O. M., \& Odaibo, B. (2004). Dockovdiacookarum infection and the prosobranch gastropod Lanisteslibycus host in Omi Stream, Ago-Iwoye, south-western Nigeria. African Journal of Biotechnology, 3(3), 202-205.

Aliu, O. O. (2006). Studies of the sediment characteristics and macroinveretebrate fauna of rivers and streams in the Opa River Basin, Ile-Ife, Osun State.M.Sc. Thesis, Dept. of Zoology, ObafemiAwolowo University, Ile-Ife.

Asibor, I. G. (2008). The Macroinvertebrate Fauna And Sediment Characteristics Of Asejire Reservoir, Southwest Nigeria. Ph.D Thesis, Dept. of Zoology, ObafemiAwolowo University, Ile-Ife, Nigeria.

Ayodele, H. A. (1979). The influence of some physico-chemical factors on the composition, abundance and character of zooplankton in some Lakes in Osun River Basin. M. Sc. Thesis, University of Ife, Ile-Ife, Nigeria.

Bidwell, E., \& Clarke, N. V. (1977).The invertebrate fauna of Lake Kainji, Nigeria. The Nigerian Field, 42(3), 104-110.

Bouchard, R. W. J. (2004). Guide to Aquatic Invertebrates of the Upper Midwest: Identification manual for students, citizen monitors and aquatic resources professionals. Water Resources Centre, USA.

Brown, D. S. (1980). Taxonomic keys to Freshwater Snails of Africa. Danish Laboratory Manual series, 7 , 25-98.

Brown, D. S. (1994).Freshwater snails of Africa and their medical importance(2nd ed.). Taylor and Francis Ltd., London.

Callisto, M., Goulart, M., Barbosa, F. A. R., \& Rocha, O. (2005). Biodiversity assessment of benthic macroinvertebrates along a reservoir cascade in the lower São Francisco river (Northeastern Brazil).Braz. J. Biol., 65(2). http://dx.doi.org/10.1590/S1519-69842005000200006

Cole, G. A. (1975). Textbook of Limnology. The C. V. Mosby Company, Saint Louis.

Cowper, S.G. (1963). Schistosomiasis in Nigeria. Annals of Tropical Medicine and Parasitology, 57, 307-322.

Edokpayi, C. A., \& Osimen, E. C. (2001). Hydrobiological studies on Ibiekuma River at Ekpoma, Southern Nigeria, after impoundment: The faunal characteristics. African Journal of Science and Technology (AJST) Science and Engineering Series, 2(1), 77-81.

Fagbola, M. S. (1989). A survey of freshwater snails in Ile-Ife for Helmith parasite.M.Sc. Thesis submitted to the Department of Zoology, ObafemiAwolowo University, Ile-Ife.

Hammer, O., Harper, D. A. T., \& Ryan, P. D. (2003). Palaeontological Statistics version 1.15. Kluwer Academic Publishers.

Harold, C., Karen, L., \& Susan, H. (1998). Organic enrichment of submarine cayon and continental shelf benthic community by macroalgal drift imported from nearshore kelp forest. Limonology and Oceanography, 43(8), 1883-1893.

Jayne, A. T., \& Joann, O.B. (1999).Substrate size selection by stream invertebrates and the influence of sand. Limnology and Oceanography, 23, 1030-1033.

Johnson, N., Revenga, C., \& Echeverria, J. (2001).Managing water for people and nature. Science, 292(5519), 1071-1072. http://dx.doi.org/10.1126/science.1058821

Koester, P., \& Bryan, C. F. (1989).Winter and spring macro-invertebrate drifts in and out pocketing of the Lower Mission River. Hydrobiologia, 185, 205-209. http://dx.doi.org/10.1007/BF00036608

Leal, J. J., \& Esteves, F. A. (1999).Density and biomass of Campsurus sp. (Ephemeroptera) and other macroinvertebrates in an Amazonian lake impacted by bauxite tailings (Lago Batata, Pará, Brazil).Amazoniana, 15(3/4), 193-209. 
Ludwig, J. A., \& Reynolds, J. F. (1988).Statistical Ecology: A primer on methods and computing. John Wiley \& Sons, New York.

Madsen, H. (1985). Keys on Tropical Freshwater Snails; Pulmonate Snails. Danish Bilharzias laboratory manual, 12 .

Mandahl-Barth, G. (1967). Revision of the African genera Potadoma Gray and Potadomoides Leloup, and description of a new species of Cleopatra (GastropodaProsobranchia: Melaniidae). Revue de Zoologies et de Botanique Afrcaines, 76, 110-131.

Mclachlam, A. J., \& Mclachlam, S. M. (1971). Benthic fauna and sediments in newly created Lake Kariba (Central Africa). Ecology, 52, 800-809. http://dx.doi.org/10.2307/1936027

Nathaniel, I. T. (2001). The Macro-invertebrate benthic fauna and bottom sediment studies of Opa Reservoir in Obafemi Awolowo University, Ile-Ife, Nigeria. M.Phil. Thesis Obafemi Awolowo University, Ile-Ife.

Ogbeibu, A. E., \& Oribhabor, B. J. (2001).The ecological impact of stream regulation using benthic macroinvertebrates as indictors. Journal of Aquatic Sciences, 16(2), 132-138. http://dx.doi.org/10.4314/jas.v16i2.20020

Oke, O. A. (1989).Studies on the macro-invertebrate benthic fauna of Owena reservoir. Hydrobiologia, 38, 241-246.

Okpalla, I. (1961). Studies on Schistosomahaemastoma infection in school children in Epe, Western Nigeria. West African Medical Journal, 10(6), 402-412.

Owojori, J. (2004). Ecology of Schistosoma intermediate snails hosts in Opa Reservoir and research ponds at Obafemi Awolowo University, Ile-Ife, Osun State. M.Sc. Thesis, Dept. of Zoology, Obafemi Awolowo University, Ile-Ife.

Rosenberg, D. M., Mccully, P., \& Pringle, C. M. (2000). Global-scale environmental effects of hydrological $\begin{array}{llll}\text { alterations: } & \text { Introduction. } & \text { Bioscience, } & \text { 746-751. }\end{array}$ http://dx.doi.org/10.1641/0006-3568(2000)050[0746:GSEEOH]2.0.CO;2

Schneider, W. (1990).FAO species identification sheets for fishery purposes. Field guide to the commercial marine resources of the Gulf of Guinea. Prepared and published with the support of the FAO Regional Office for Africa. Rome, FAO.

Taiwo, S. A. (1983). Studies on the Benthic fauna of Kainji Lake, Nigeria. Annual report of Lake Kainji Research Project, New Bussa, 3, 33-36.

Torloni, C. E. (1994). Management of fishing resources in CESPs reservoirs. In F. A. R. Barbosa (Ed.), Workshop: Brazilian Programme on Conservation and Management of Inland Waters. ActaLimnol. Brasil, 5, 177-186.

Tundisi, J. G., \& Straškraba, M. (1999).Theoretical reservoir ecology and its applications. Backhuys Publishers, The Netherlands.

Verma, P. S. (2006). A manual of practical zoology invertebrates. S. Chand \& Company Ltd, New Delhi.

\section{Copyrights}

Copyright for this articleis retained by the author(s), with first publication rights granted to the journal.

This is an open-access article distributed under the terms and conditions of the CreativeCommons Attribution license (http://creativecommons.org/licenses/by/3.0/). 